\title{
Representations of the solvable group acting on a homogeneous Siegel domain
}

\author{
By Hideyuki IsHI \\ Department of Mathematics, Kyoto University
}

1. Introduction. Let $(\mathfrak{g}, j, \omega)$ be a normal $j$-algebra and $G$ the solvable Lie group corresponding to $\mathfrak{g}$. As is shown by Piatetskii-Shapiro [12], $G$ is realized as an affine transformation group of a homogeneous Siegel domain $D$, so that one can define representations of $G$ on the space of holomorphic functions on $D$ in a natural way (see (4.1)). Our concern is to get unitary representations of $G$ embedded in these representations. A similar situation for the case of symmetric Siegel domains has been investigated by Vergne and Rossi [15] (see also Wallach [17] and Clerc [3]) about the analytic continuation of the holomorphic discrete series representation of a semisimple Lie group. In the present paper, we determine all of such unitary representations of $G$ by relating them to the positivity of certain invariant distributions (Riesz distributions) on the dual cone and classify them by using the theory of orbit method.

2. Preliminaries. We first recall the definition of normal $j$-algebras. Let $\mathfrak{g}$ be a real split solvable Lie algebra, $j$ a linear operator on $\mathfrak{g}$ such that $j^{2}=-\mathrm{id} \mathfrak{g}$, and $\omega$ a linear form on $\mathfrak{g}$. The triple $(\mathfrak{g}, j, \omega)$ is called a normal $j$-algebra if the following two conditions are satisfied:

(i) $\left[Y_{1}, Y_{2}\right]+j\left[j Y_{1}, Y_{2}\right]+j\left[Y_{1}, j Y_{2}\right]-\left[j Y_{1}, j Y_{2}\right]=0$ for all $Y_{1}, Y_{2} \in \mathfrak{g}$,

(ii) a bilinear form $\left(Y_{1} \mid Y_{2}\right)_{\omega}:=\omega\left(\left[Y_{1}, j Y_{2}\right]\right)$ defines a $j$-invariant inner product on $\mathfrak{g}$.

Let $\mathfrak{a}$ be the orthogonal complement of the subspace $[\mathfrak{g}, \mathfrak{g}] \subset \mathfrak{g}$ with respect to the inner product $(\cdot \mid \cdot)_{\omega}$. Then $\mathfrak{a}$ is a commutative subalgebra of $\mathfrak{g}$. Put $r:=\operatorname{dim} \mathfrak{a}$, and for a linear form $\alpha \in \mathfrak{a}^{*}$, set $\mathfrak{g}_{\alpha}:=\{Y \in \mathfrak{g} ;[C, Y]=\alpha(C) Y$ for all $C \in \mathfrak{a}\}$. The following proposition is fundamental for our study.

Proposition 1 ([12, Chapter 2, Sections 3 and 5]). (i) There is a linear basis $\left\{A_{1}, \ldots, A_{r}\right\}$ of $\mathfrak{a}$ such that if one puts $E_{l}:=-j A_{l}$, then $\left[A_{k}, E_{l}\right]=\delta_{k l} E_{l}(1 \leq$ $k, l \leq r)$. 
(ii) Let $\alpha_{1}, \ldots, \alpha_{r}$ be the basis of $\mathfrak{a}^{*}$ dual to $A_{1}, \ldots, A_{r}$. Then one has a decomposition $\mathfrak{g}=\mathfrak{g}(1) \oplus \mathfrak{g}(1 / 2) \oplus \mathfrak{g}(0)$ with

$$
\begin{aligned}
& \mathfrak{g}(1):=\sum_{k=1}^{r} \mathbb{R} E_{k} \oplus \sum_{1 \leq k<m \leq r}^{\oplus} \mathfrak{g}_{\left(\alpha_{m}+\alpha_{k}\right) / 2}, \quad \mathfrak{g}(1 / 2):=\sum_{k=1}^{r} \oplus \mathfrak{g}_{\alpha_{k} / 2}, \\
& \mathfrak{g}(0):=\mathfrak{a} \oplus \sum_{1 \leq k<m \leq r}^{\oplus} \mathfrak{g}_{\left(\alpha_{m}-\alpha_{k}\right) / 2} .
\end{aligned}
$$

(iii) One has $[\mathfrak{g}(\mu), \mathfrak{g}(\nu)] \subset \mathfrak{g}(\mu+\nu) \quad(\mu, \nu=0,1 / 2,1)$, where $\mathfrak{g}(\mu):=\{0\}$ if $\mu>1$.

The assertion (iii) tells us that the subspace $\mathfrak{g}(0)$ is a subalgebra of $\mathfrak{g}$ and that the corresponding Lie group $H:=\exp \mathfrak{g}(0)$ acts on $\mathfrak{g}(1 / 2)$ and $\mathfrak{g}(1)$ respectively by the adjoint actions. Put $E:=E_{1}+\cdots+E_{r} \in \mathfrak{g}(1)$ and let $\Omega$ be the $H$-orbit through $E$. Then $\Omega$ is a regular open convex cone in $\mathfrak{g}(1)$ and $H$ acts on $\Omega$ simply transitively. The linear operator $j$ preserves the subspace $\mathfrak{g}(1 / 2)$, so that $j$ defines a complex structure on $\mathfrak{g}(1 / 2)$. We define a $\mathfrak{g}(1)_{\mathbb{C}^{-v a l u e d}}$ Hermitian map $Q$ on $\mathfrak{g}(1 / 2)$ by $Q\left(u, u^{\prime}\right):=\left(\left[j u, u^{\prime}\right]+i\left[u, u^{\prime}\right]\right) / 4\left(u, u^{\prime} \in \mathfrak{g}(1 / 2)\right)$. Then $Q$ is $\Omega$-positive, that is, $Q(u, u) \in \bar{\Omega} \backslash\{0\}$ for all $u \in \mathfrak{g}(1 / 2) \backslash\{0\}$. Now we define the Siegel domain $D$ corresponding to $(\mathfrak{g}, j, \omega)$ by $D:=\left\{(z, u) \in \mathfrak{g}(1)_{\mathbb{C}} \times \mathfrak{g}(1 / 2) ; \operatorname{Im} z-Q(u, u) \in \Omega\right\}$.

By Proposition 1 (iii), the space $\mathfrak{n}(Q):=\mathfrak{g}(1) \oplus \mathfrak{g}(1 / 2)$ is a nilpotent subalgebra of $\mathfrak{g}$. Put $N(Q):=\exp \mathfrak{n}(Q)$. Then the solvable Lie group $G$ is a semidirect product $N(Q) \rtimes H$. We realize $G$ as an affine transformation group on $\mathfrak{g}(1)_{\mathbb{C}} \times \mathfrak{g}(1 / 2)$ by setting $g_{0} \cdot(z, u):=\left(t_{0} \cdot z+x_{0}+2 i Q\left(t_{0} \cdot u, u_{0}\right)+i Q\left(u_{0}, u_{0}\right), t_{0} \cdot u+u_{0}\right)$ for $g_{0}=\exp \left(x_{0}+u_{0}\right) t_{0} \in G\left(x_{0} \in \mathfrak{g}(1), u_{0} \in \mathfrak{g}(1 / 2), t_{0} \in H\right)$ and $(z, u) \in \mathfrak{g}(1)_{\mathbb{C}} \times \mathfrak{g}(1 / 2)$. Then $G$ acts on $D$ simply transitively.

For $s=\left(s_{1}, \ldots, s_{r}\right) \in \mathbb{C}^{r}$, let $\chi_{s}$ be the one-dimensional representation of $G$ such that $\chi_{s}\left(\exp \left(\sum_{k=1}^{r} c_{k} A_{k}\right)\right)=e^{s_{1} c_{1}+\cdots+s_{r} c_{r}}\left(c_{1}, \ldots, c_{r} \in \mathbb{R}\right)$. We define a function $\Delta_{s}$ on $\Omega$ by $\Delta_{s}(t \cdot E):=\chi_{s}(t)(t \in H)$ and extend it to the domain $\Omega+i \mathfrak{g}(1) \subset \mathfrak{g}(1)_{\mathbb{C}}$ by analytic continuation.

3. Riesz distributions on the dual cone. Let $\Omega^{*}$ be the dual cone of $\Omega$, that is, $\Omega^{*}:=\left\{\xi \in \mathfrak{g}(1)^{*} ;\langle x, \xi\rangle>0\right.$ for all $\left.x \in \bar{\Omega} \backslash\{0\}\right\}$. The group $H$ acts on $\Omega^{*}$ simply transitively by the contragredient action. For $\varepsilon=\left(\varepsilon_{1}, \ldots, \varepsilon_{r}\right) \in\{0,1\}^{r}$, let $E_{\varepsilon}^{*}$ be a linear form on $\mathfrak{g}(1)$ given by $\left\langle\sum_{k=1}^{r} x_{k k} E_{k}+\sum_{m>k} X_{m k}, E_{\varepsilon}^{*}\right\rangle:=\sum_{k=1}^{r} \varepsilon_{k} x_{k k}$ for $x_{k k} \in \mathbb{R}$ and $X_{m k} \in \mathfrak{g}_{\left(\alpha_{m}+\alpha_{k}\right) / 2}$. Let $\mathcal{O}_{\varepsilon}^{*}$ be the $H$-orbit in $\mathfrak{g}(1)^{*}$ through $E_{\varepsilon}^{*}$. Then the $H$-orbit decomposition of the closure of the cone $\Omega^{*}$ is given as

$$
\overline{\Omega^{*}}=\bigsqcup_{\varepsilon \in\{0,1\}^{r}} \mathcal{O}_{\varepsilon}^{*}
$$


Now we consider tempered distributions $\mathcal{R}_{s}^{*}\left(s \in \mathbb{C}^{r}\right)$ on $\mathfrak{g}(1)^{*}$ characterized by the Laplace transform:

$$
\left\langle\mathcal{R}_{s}^{*}, e^{-\langle y, \cdot\rangle}\right\rangle=\Delta_{-s^{*}}(y) \quad(y \in \Omega),
$$

where $s^{*}:=\left(s_{r}, \ldots, s_{1}\right) \in \mathbb{C}^{r}$. These $\mathcal{R}_{s}^{*}$ are relatively invariant under $H$ and supported by $\overline{\Omega^{*}}$. We call $\mathcal{R}_{s}^{*}$ the Riesz distributions on $\Omega^{*}$ (see [7] for the precise definition of the Riesz distributions).

The results of [8] tell us that the structure of the positive Riesz distributions is closely related to the orbit structure (3.1) of $\overline{\Omega^{*}}$. Put $q_{k}(\varepsilon):=\sum_{m>k} \varepsilon_{m} \operatorname{dim} \mathfrak{g}_{\left(\alpha_{m}-\alpha_{k}\right) / 2}$ $(k=1, \ldots, r)$,

$$
\mathfrak{X}(\varepsilon):=\left\{s \in \mathbb{C}^{r} ; \operatorname{Re} s_{k}>q_{k}(\varepsilon) / 2\left(\text { if } \varepsilon_{k}=1\right), \quad \operatorname{Re} s_{k}=q_{k}(\varepsilon) / 2\left(\text { if } \varepsilon_{k}=0\right)\right\},
$$

and $\mathfrak{X}:=\bigsqcup_{\varepsilon \in\{0,1\}^{r}} \mathfrak{X}(\varepsilon)$. For $s \in \mathbb{C}^{r}$, we set $\operatorname{Re} s^{*}:=\left(\operatorname{Re} s_{r}, \ldots, \operatorname{Re} s_{1}\right)$.

Proposition 2. (i) The Riesz distribution $\mathcal{R}_{\operatorname{Re} s^{*}}^{*}$ is positive if and only if $s \in \mathfrak{X}$. (ii) When $s \in \mathfrak{X}(\varepsilon)$, the distribution $\mathcal{R}_{\operatorname{Re} s^{*}}^{*}$ is an $H$-relatively invariant positive measure on $\mathcal{O}_{\varepsilon}^{*}$.

4. Representations of $G$ on spaces of holomorphic functions on $D$. Let $\mathcal{H}(D)$ be the space of holomorphic functions on $D$. For $s \in \mathbb{C}^{r}$, we define a representation $\pi_{s}$ on $\mathcal{H}(D)$ by

$$
\pi_{s}(g) F(p):=\chi_{-s / 2}(g) F\left(g^{-1} \cdot p\right) \quad(g \in G, p \in D, F \in \mathcal{H}(D)) .
$$

We consider the subspace $\mathcal{H}_{s}(D)$ of $\mathcal{H}(D)$ satisfying the following two conditions:

(i) $\mathcal{H}_{s}(D)$ has a Hilbert space structure with reproducing kernel,

(ii) $\left(\pi_{s}, \mathcal{H}_{s}(D)\right)$ is a unitary representation of $G$.

The condition (i) is equivalent to the statement that the Hilbert space $\mathcal{H}_{s}(D)$ is continuously embedded into a topological vector space $\mathcal{H}(D)$ with the compact open topology (see [3]). We note that non-zero $\mathcal{H}_{s}(D)$ is unique, though it might not exist for some $s \in \mathbb{C}^{r}$.

Proposition 3. If non-zero $\mathcal{H}_{s}(D)$ exists, the reproducing kernel $K^{s}$ of $\mathcal{H}_{s}(D)$ is given (up to a positive constant) by

$$
K^{s}\left((z, u),\left(z^{\prime}, u^{\prime}\right)\right)=\Delta_{-\operatorname{Re} s}\left(\left(z-\bar{z}^{\prime}\right) / i-2 Q\left(u, u^{\prime}\right)\right) \quad\left((z, u),\left(z^{\prime}, u^{\prime}\right) \in D\right) .
$$

By Proposition 3 and the argument given in [15, Chapter 3], we see that the non-vanishing of the Hilbert space $\mathcal{H}_{s}(D)$ is equivalent to the fact that the function $\Delta_{-\operatorname{Re} s}$ on $\Omega$ is of positive type. On the other hand, a Bochner type theorem [4, Satz 5.1] states that a homogeneous function $f$ on $\Omega$ is of positive type if and only 
if $f$ is the Laplace transform of a positive measure on $\overline{\Omega^{*}}$. Therefore (3.2) and Proposition 2 (i) lead us to the following theorem.

Theorem 4. Non-zero $\mathcal{H}_{s}(D)$ exists if and only if $s \in \mathfrak{X}$.

In view of Theorem 4 and the result of [15], the set $\mathfrak{X}$ can be regarded as a non-symmetric analogue of the Wallach sets for symmetric Siegel domains.

Next we describe each $\mathcal{H}_{s}(D)(s \in \mathfrak{X})$ through the Fourier-Laplace transformation. For $\xi \in \overline{\Omega^{*}}$, put $Q_{\xi}:=2 \xi \circ Q$ and $N_{\xi}:=\left\{v \in \mathfrak{g}(1 / 2) ; Q_{\xi}(v, v)=0\right\}$. Then $Q_{\xi}$ induces a positive definite Hermitian form on $\mathfrak{g}(1 / 2) / N_{\xi}$. Let $\mathcal{F}_{\xi}$ be the space of holomorphic functions $\phi$ on $\mathfrak{g}(1 / 2)$ such that

(i) $\phi(u+v)=\phi(u)$ for all $u \in \mathfrak{g}(1 / 2)$ and $v \in N_{\xi}$,

(ii) $\|\phi\|_{\xi}^{2}:=\int_{\mathfrak{g}_{(1 / 2) / N_{\xi}}}|\phi(u)|^{2} e^{-Q_{\xi}(u, u)} d m_{\xi}(\dot{u})<\infty$,

where $d m_{\xi}$ is the Lebesgue measure on $\mathfrak{g}(1 / 2) / N_{\xi}$ normalized in such a way that $\int_{\mathfrak{g}_{(1 / 2) / N_{\xi}}} e^{-Q_{\xi}(u, u)} d m_{\xi}(\dot{u})=1$. In other words, $\mathcal{F}_{\xi}$ is the Fock space on $\mathfrak{g}(1 / 2) / N_{\xi}$ with reproducing kernel $e^{Q_{\xi}(\cdot, \cdot)}$ (see [1]). Keeping Proposition 2 (ii) in mind, we define $\mathcal{L}_{s}(s \in \mathfrak{X}(\varepsilon))$ to be the space of measurable functions $f$ on $\mathcal{O}_{\varepsilon}^{*} \times \mathfrak{g}(1 / 2)$ such that

(i) $f(\xi, \cdot) \in \mathcal{F}_{\xi}$ for almost all $\xi \in \mathcal{O}_{\varepsilon}^{*}$ with respect to the measure $d \mathcal{R}_{\operatorname{Re} s^{*}}^{*}$,

(ii) $\|f\|^{2}:=\int_{\mathcal{O}_{\varepsilon}^{*}}\|f(\xi, \cdot)\|_{\xi}^{2} d \mathcal{R}_{\operatorname{Re} s^{*}}^{*}(\xi)<\infty$.

Then $\mathcal{L}_{s}$ forms a Hilbert space, realizing the direct integral $\int_{\mathcal{O}^{*}}^{\oplus} \mathcal{F}_{\xi} d \mathcal{R}_{\operatorname{Re} s^{*}}^{*}(\xi)$.

Theorem 5. For $s \in \mathfrak{X}(\varepsilon)$, one has the unitary isomorphism $\Phi_{s}: \mathcal{L}_{s} \ni f \mapsto F \in$ $\mathcal{H}_{s}(D)$ given by

$$
F(z, u):=\int_{\mathcal{O}_{\varepsilon}^{*}} e^{i\langle z, \xi\rangle} f(\xi, u) d \mathcal{R}_{\operatorname{Re} s^{*}}^{*}(\xi) \quad((z, u) \in D) .
$$

We define the so-called Fock representation $\tau_{\xi}$ of $N(Q)$ on $\mathcal{F}_{\xi}$ by $\tau_{\xi}\left(\exp \left(x_{0}+u_{0}\right)\right) \phi(u):=e^{-i\left\langle x_{0}, \xi\right\rangle+Q_{\xi}\left(u, u_{0}\right)-Q_{\xi}\left(u_{0}, u_{0}\right) / 2} \phi\left(u-u_{0}\right) \quad\left(u, u_{0} \in \mathfrak{g}(1 / 2), x_{0} \in \mathfrak{g}(1)\right)$ and a unitary representation $\check{\pi}_{s}(s \in \mathfrak{X}(\varepsilon))$ of $G$ on $\mathcal{L}_{s}$ by

$$
\begin{array}{r}
\check{\pi}_{s}(t) f(\xi, u):=\chi_{\bar{s} / 2}(t) f\left(t^{-1} \cdot \xi, t^{-1} \cdot u\right) \quad(t \in H), \\
\check{\pi}_{s}(n) f(\xi, \cdot):=\tau_{\xi}(n) f(\xi, \cdot) \quad(n \in N(Q)),
\end{array}
$$

where $\bar{s}:=\left(\bar{s}_{1}, \ldots, \bar{s}_{r}\right) \in \mathbb{C}^{r}$.

Proposition 6. The operator $\Phi_{s}: \mathcal{L}_{s} \rightarrow \mathcal{H}_{s}(D)$ is an intertwining operator between $\check{\pi}_{s}$ and $\pi_{s}$. In particular, $\Phi_{s}$ induces the direct integral decomposition of $\left.\pi_{s}\right|_{N(Q)}:$

$$
\left.\left.\pi_{s}\right|_{N(Q)} \simeq \int_{\mathcal{O}_{\varepsilon}^{*}}^{\oplus} \tau_{\xi}\right|_{N(Q)} d \mathcal{R}_{\operatorname{Re} s^{*}}^{*}(\xi)
$$


5. The orbit method and the classification of $\pi_{\boldsymbol{s}}$. Since $\mathcal{H}_{s}(D)$ has a reproducing kernel, we obtain the following proposition from Kunze's result [11]:

Proposition 7. The unitary representation $\left(\pi_{s}, \mathcal{H}_{s}(D)\right)(s \in \mathfrak{X})$ of $G$ is irreducible.

On the other hand, since $G$ is exponential solvable, the equivalence classes of IUR's (irreducible unitary representations) of $G$ are classified by the orbit method. Namely, there is a bijection, called the Kirillov-Bernat correspondence, between elements of the unitary dual $\hat{G}$ and coadjoint orbits in $\mathfrak{g}^{*}$ (see [2] for this). In this section, we shall describe the coadjoint orbits in $\mathfrak{g}^{*}$ corresponding to our $\left(\pi_{s}, \mathcal{H}_{s}(D)\right)$, so that the classification of these IUR's is obtained.

We first identify each $\zeta=\left(\zeta_{1}, \ldots, \zeta_{r}\right) \in \mathbb{R}^{r}$ with the linear form on $\mathfrak{g}$ given by $\left\langle\sum_{k=1}^{r} c_{k} A_{k}+Y, \zeta\right\rangle:=\sum_{k=1}^{r} \zeta_{k} c_{k} \quad\left(c_{1}, \ldots, c_{r} \in \mathbb{R}, Y \in[\mathfrak{g}, \mathfrak{g}]\right)$, and for $\varepsilon \in\{0,1\}^{r}$, we set $Z(\varepsilon):=\left\{\zeta \in \mathfrak{g}^{*} ; \zeta_{k}=0\right.$ for all $k$ such that $\left.\varepsilon_{k}=1\right\}$. Let $\mathfrak{h}_{\xi}\left(\xi \in \mathfrak{g}(1)^{*}\right)$ denote the Lie algebra of the stabilizer $H_{\xi}$ at $\xi$ in $H$.

Proposition 8. Let $\zeta \in Z(\varepsilon)$ with $\varepsilon \in\{0,1\}^{r}$. Then the coadjoint orbit in $\mathfrak{g}^{*}$ through $-E_{\varepsilon}^{*}+\zeta$ is described as

$$
\operatorname{Ad}^{*}(G)\left(-E_{\varepsilon}^{*}+\zeta\right)=\bigsqcup_{\xi \in \mathcal{O}_{\varepsilon}^{*}}\left(-\xi+\zeta+\left(\mathfrak{g}(1) \oplus N_{\xi} \oplus \mathfrak{h}_{\xi}\right)^{\perp}\right)
$$

where $\left(\mathfrak{g}(1) \oplus N_{\xi} \oplus \mathfrak{h}_{\xi}\right)^{\perp}:=\left\{\eta \in \mathfrak{g}^{*} ;\langle Y, \eta\rangle=0\right.$ for all $\left.Y \in \mathfrak{g}(1) \oplus N_{\xi} \oplus \mathfrak{h}_{\xi}\right\}$. Distinct $(\varepsilon, \zeta)$ 's give distinct orbits.

We note that the coadjoint orbit through $\zeta \in Z(0, \ldots, 0)=\mathbb{R}^{r}$ is a one-point set $\{\zeta\}$. On the other hand, the coadjoint orbit through $-E_{(1, \ldots, 1)}^{*}$ is the open set $-\Omega^{*}+\mathfrak{g}(1 / 2)^{*}+\mathfrak{g}(0)^{*}$ in $\mathfrak{g}^{*}$.

Let $\Theta(\varepsilon, \zeta)$ be the set given by

$$
\left.\Theta(\varepsilon, \zeta):=\left\{s \in \mathbb{C}^{r} ; \operatorname{Re} s_{k}>q_{k}(\varepsilon) / 2 \text { (if } \varepsilon_{k}=1\right), \quad s_{k}=q_{k}(\varepsilon) / 2-2 i \zeta_{k}\left(\text { if } \varepsilon_{k}=0\right)\right\} .
$$

Then we have $\mathfrak{X}(\varepsilon)=\bigsqcup_{\zeta \in Z(\varepsilon)} \Theta(\varepsilon, \zeta)$, so that Theorem 4 implies that $\mathcal{H}_{s}(D) \neq\{0\}$ exists if and only if $s$ belongs to some $\Theta(\varepsilon, \zeta)$.

Theorem 9. (i) If $s \in \Theta(\varepsilon, \zeta)$, then the IUR $\left(\pi_{s}, \mathcal{H}_{s}(D)\right)$ of $G$ corresponds to the coadjoint orbit through $-E_{\varepsilon}^{*}+\zeta \in \mathfrak{g}^{*}$ by the Kirillov-Bernat correspondence.

(ii) Two IUR's $\left(\pi_{s}, \mathcal{H}_{s}(D)\right)$ and $\left(\pi_{s^{\prime}}, \mathcal{H}_{s^{\prime}}(D)\right)$ are equivalent if and only if $s$ and $s^{\prime}$ belong to the same $\Theta(\varepsilon, \zeta)$.

When $s=\left(-2 i \zeta_{1}, \ldots,-2 i \zeta_{r}\right) \in \Theta(0, \zeta)$, it can be checked directly that $\mathcal{H}_{s}(D)$ equals the space of constant functions on $D$ and the representation $\pi_{s}$ is equivalent to the unitary character $\chi_{i \zeta}$. We see from Theorem 9 that this IUR $\pi_{s}$ corresponds 
to the one-point set $\{\zeta\}=\operatorname{Ad}^{*}(G) \zeta$. On the other hand, Theorem 9 also states that the unitary representations of $G$ realized naturally on the various weighted Bergman spaces on $D$ investigated by Gindikin [7] (see also Rossi and Vergne [14]) correspond to the same open orbit $\operatorname{Ad}^{*}(G)\left(-E_{(1, \ldots, 1)}^{*}\right)$ mentioned above, so that all of such representations are equivalent.

The details [10] will appear elsewhere.

\section{References}

[1] B. Bargmann, On a Hilbert space of analytic functions and an associated integral transform I, Comm. Pure Appl. Math., 14 (1961), 187-214.

[2] P. Bernat et al., Représentations des groupes de Lie résolubles, Dunod, Paris, 1972.

[3] J. -L. Clerc, Laplace transform and unitary highest weight modules, J. Lie Theory, 5 (1995), 225-240.

[4] J. Dorfmeister und M. Koecher, Reguläre Kegel, Jahresber. Deutsch. Math. Verein. 81 (1979), 109-151.

[5] J. Faraut and A. Korányi, Analysis on symmetric cones, Oxford Mathematical Monographs, Clarendon Press, Oxford, 1994.

[6] S. G. Gindikin, Analysis in homogeneous domains, Russian Math. Surveys, 19 (1964), 1-89.

[7] - , Invariant generalized functions in homogeneous domains, Funct. Anal. Appl., 9 (1975), 50-52.

[8] H. Ishi, Positive Riesz distributions on homogeneous cones, J. Math. Soc. Japan, to appear.

[9] —, An explicit description of positive Riesz distributions on homogeneous cones, Proc. Japan Acad., 74 (1998), 132-134.

[10] -, Representations of the affine transformation groups acting simply transitively on Siegel domains, preprint, 1998.

[11] R. A. Kunze, On the irreducibility of certain multiplier representations, Bull. Amer. Math. Soc., 68 (1962), 93-94.

[12] I. I. Piatetskii-Shapiro, Automorphic functions and the geometry of classical domains, Gordon and Breach, New York, 1969.

[13] M. Riesz, L'intégrale de Riemann-Liouville et le problème de Cauchy, Acta Math., 81 (1949), 1-223.

[14] H. Rossi and M. Vergne, Representations of certain solvable Lie groups on Hilbert spaces of holomorphic functions and the application to the holomorphic discrete series of a semisimple Lie group, J. Funct. Anal., 13 (1973), 324-389.

[15] M. Vergne and H. Rossi, Analytic continuation of the holomorphic discrete series of a semi-simple Lie group, Acta Math., 136 (1976), 1-59.

[16] E. B. Vinberg, The theory of convex homogeneous cones, Trans. Moscow Math. Soc., 12 (1963), 340-403.

[17] N. Wallach, The analytic continuation of the discrete series, I, II, Trans. Amer. Math. Soc., 251 (1979), 1-17; ibid., 19-37. 